\title{
SIMPLE MODELS FOR DEMONSTRATION OF FLOW OF GROUND-WATER ${ }^{1}$ )
}

\author{
W. R. VAN WIJK \\ Lab. of Physics and Meteorology of the Agricultural University, \\ Wageningen, Holland
}

Models for demonstration of the motion of water through porous materials are often used. They are of great value for instruction and may serve to obtain approximate quantitative indications about the resistance of the soil to the flow of ground-water. Particularly instructive are models in which the

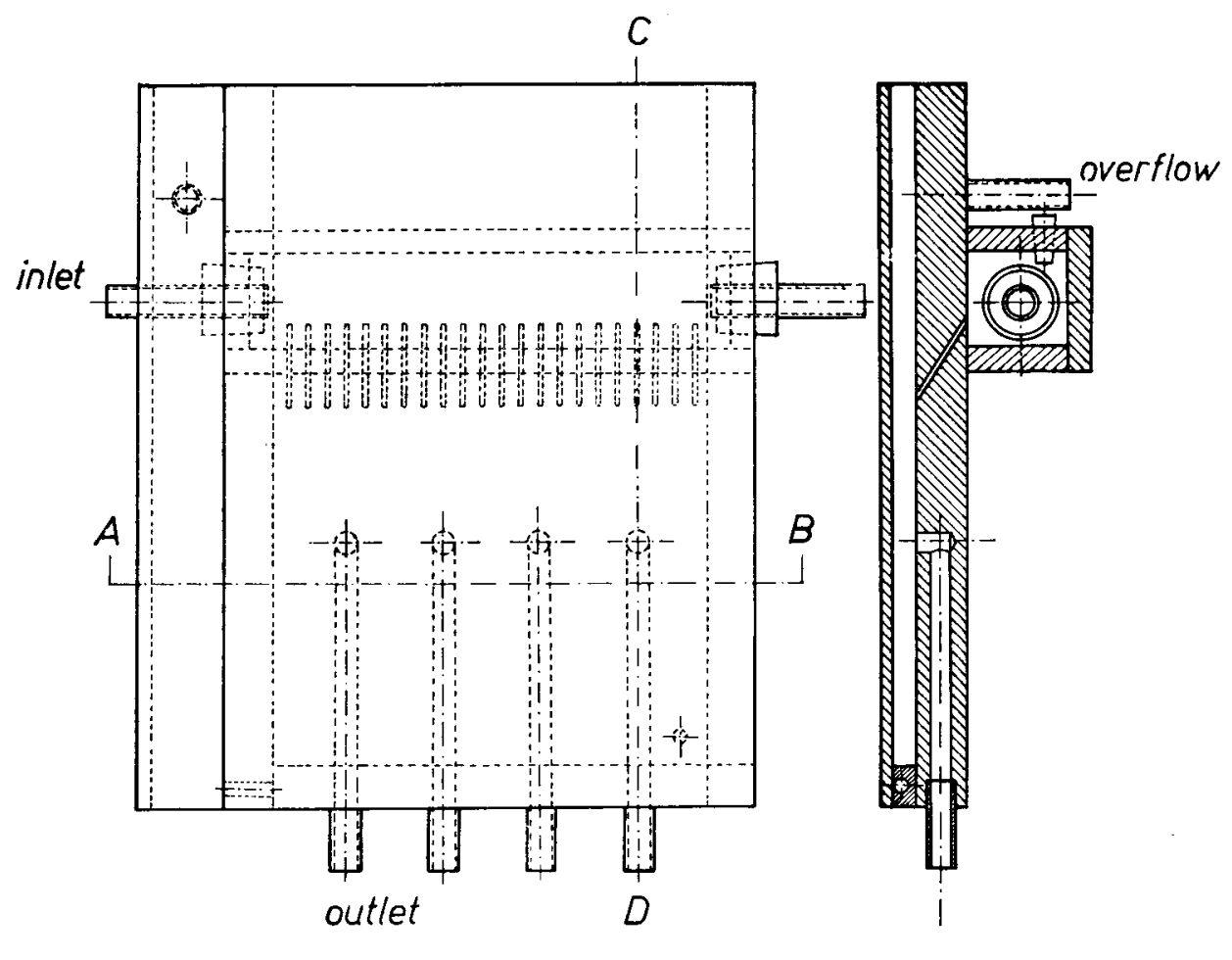

section $C-D$

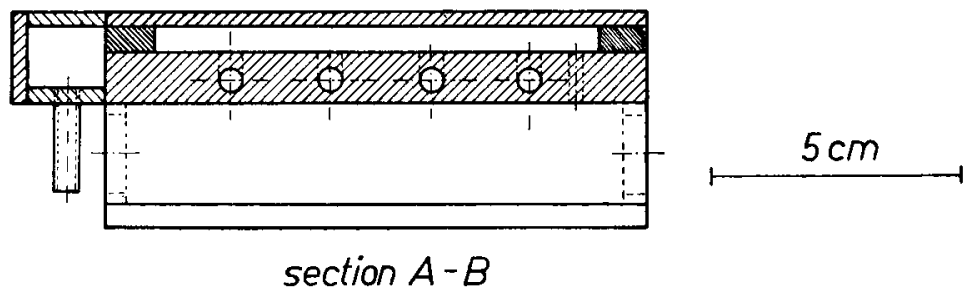

Fig. 1 Perspex cunette for projection of flow-patterns in drainage.

1) Received for publication June 19, 1957. 
lines of flow are made visible by adding colouring substances such as ink or potassium permanganate to the liquid. If two different liquids are used in the model, one being colourless and the other coloured, the model is not easy to operate and it takes a considerable time to change from one flow pattern to another, since the flow of two liquids must be adjusted.

In the laboratory of physics and meteorology at Wageningen a kind of model has recently been developed which allows quick changing from one flow pattern to another. The models are even used in the same way as lanternslides. In a quick succession different flow patterns are projected on a screen during a classroom lecture.

The model consists of a perspex cuvette which is placed before the condensor of a projection-lantern. In the cuvette a plate can be inserted which by variation of its thickness is used to imitate the different resistances occurring in the soil. A drawing of the cuvette is given in figure 1.

Water entering the cuvette leaves it through a number of small holes in the front wall. This cuvette was designed for demonstration of flow of groundwater to drain tubes. Other types of cuvettes are also used; for instance the out-flow of water may take place through a large number of holes placed in a row at the bottom of the cuvette. Such a cuvette is used to study the deformation of the streamlines caused by solid bodies placed in an originally parallel flow.

The variable part of the model consists of the rectangular plate, also of perspex, which can be inserted in the cuvette and which is carefully milled to the proper profile.

In figure 2, a plate is shown which blocks off flow entirely below the outlet holes in the cuvette. This imitates an impermeable layer below the drain-tubes. A layer of reduced permeability is imitated by a small distance between the plate and the wall of the cuvette.

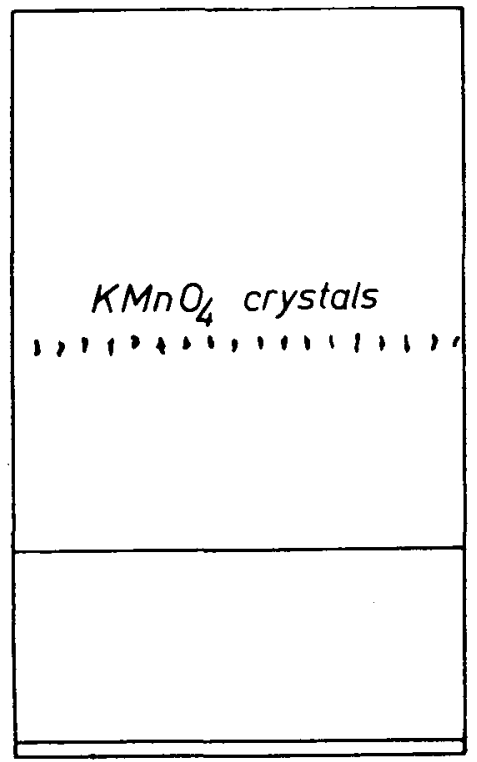

Fig. 2 Plate used for the demonstraTION OF DRAINAGE IN CASE OF A NON PERMEABLE LAYER CLOSELY BEI.OW THE DRAIN TUBES. 


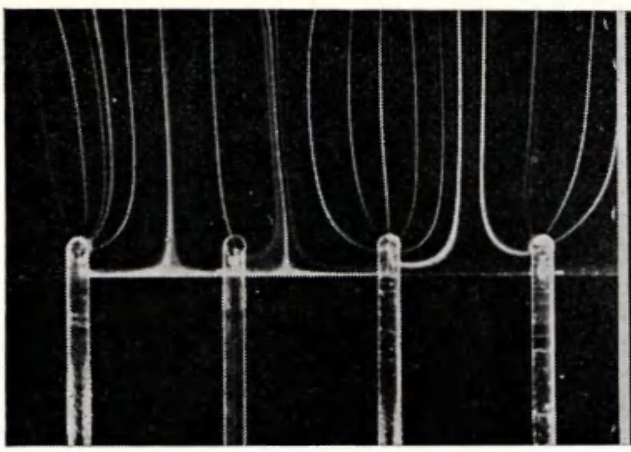

Fig. 3 Drain tubes above an impermeABLE LAYER.

An air bubble in the drain tube second from the left is restricting the flow through it.

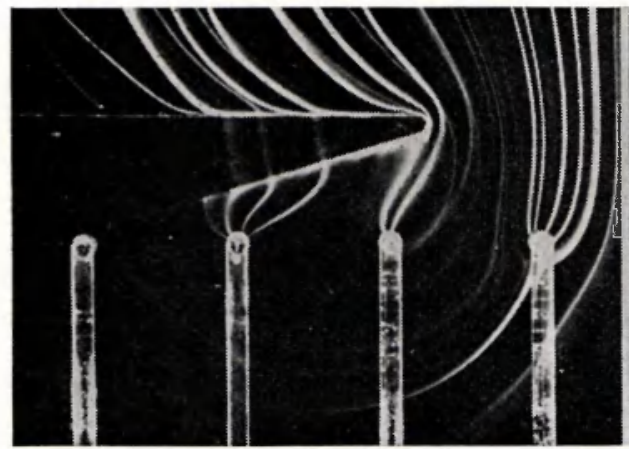

Fig. 5 Wedgeformed LAYER OF REDUCED PERMEABILITY ABOVE DRAIN TUBES.

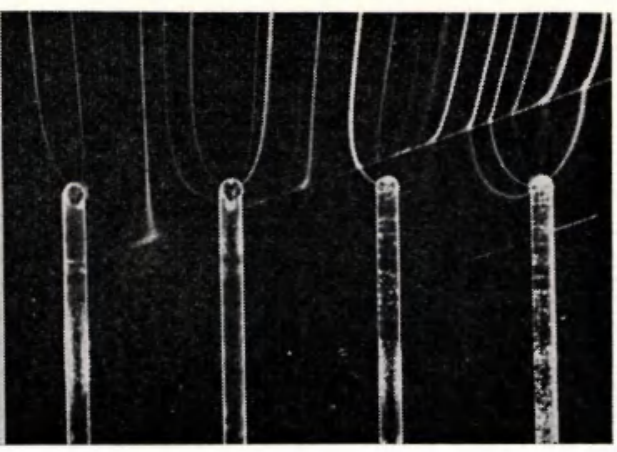

Fig. 4 Orlique layer of reduced perMEABILITY.

Two drain tubes are in the layer of low permeability.

Note the refraction of the lines of flow at the boundary.

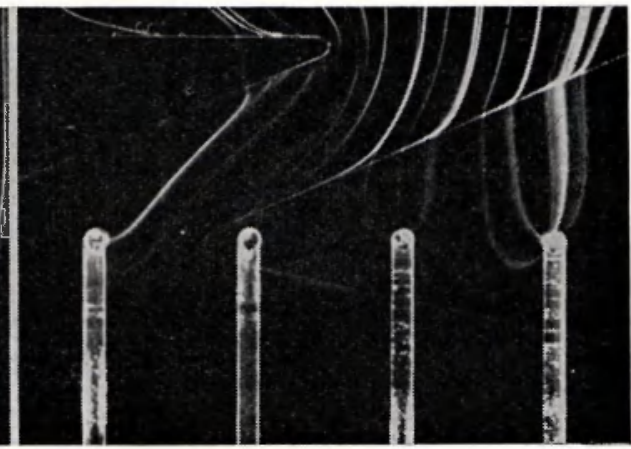

Fig. 6 TWO WEDGEFORMED LAYERS OF LOW PERMEABILITY.

Three drain tubes are in the "wedge" right hand.

Near the top of the plate a row of small crystals of potassium permanganate are glued to it with a water insoluble glue. Water flowing past the crystals obtains an intense red colour. No adjusting of the flow of water through the cuvette is necessary after insertment of the plate, though the increased resistance in the cuvette causes a slower out-flow but the excess of incoming water leaves the cuvette through the over-flow pipe near the top (fig. 1). This makes a quick interchange of the plates possible. After insertion of a new plate one has only to waite a few seconds for the turbulence caused by the motion of the plate to disappear. The models can be projected one after the other with approximately half a minute interval.

Some photographs of the projected models are shown in the figures 3,4 , 5 , and 6 . They pertain to drainage.

A great advantage of the use of a model as compared with a lantern-slide is that one can vary the conditions of flow during the projection. For instance 


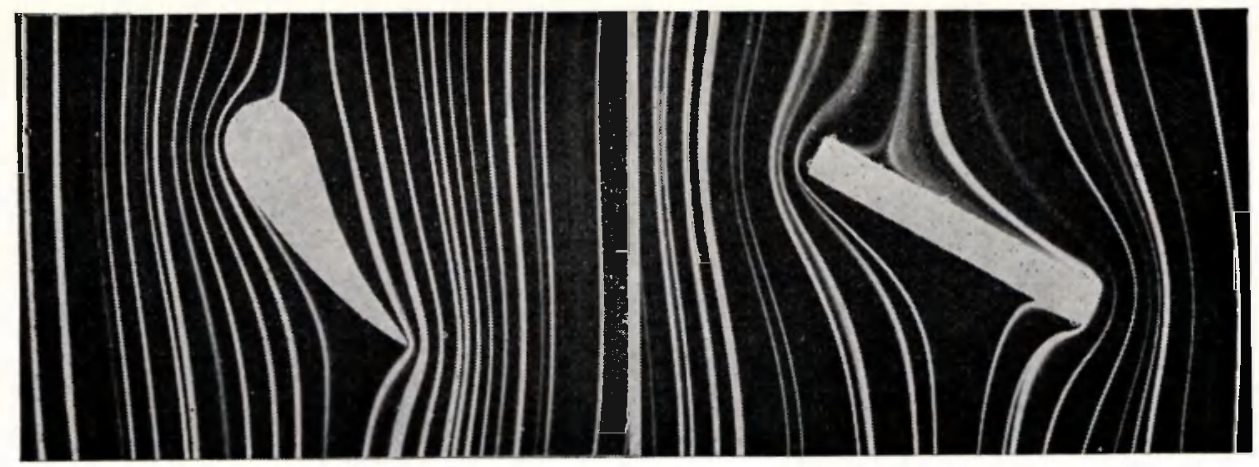

Fig. 7 Wing profiLe of aERoplane.

Fig. 8 Slab inClined to Lines of FLow.

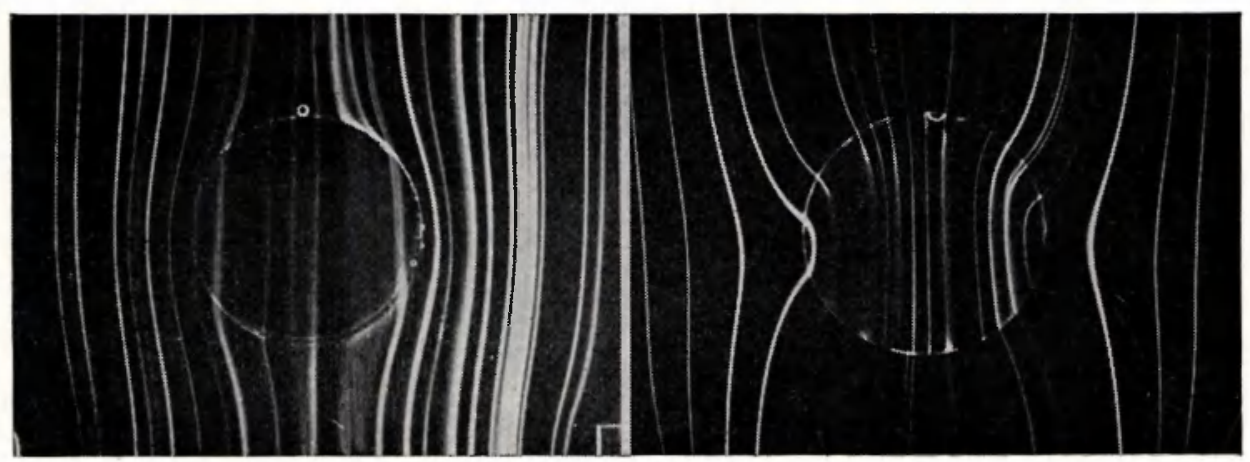

Fig. 9 SPhene of LoWer PEnmeability iN FIELD OF FLOW.

This is a model of a diamagnetic sphere in a homogeneous magnetic field.
Fig. 10 Sphere of higher Permeability IN FIELD OF FLOW.

This is a model of a paramagnetic sphere in a homogeneous magnetic field.

one of the drain tubes can be given a higher resistance (fouling) by simply squeezing the rubber tube through which the water flows out of one of the openings in the cuvette. It is highly instructive to observe how the original pattern is rapidly restored after the "fouled" drain-pipe has been cleaned.

In figures $7,8,9$, and 10 models are shown of the distortion of flow by solid bodies. Here again variation of the conditions of flow is easily made by increasing the resistance in a part of the outlet holes by squeezing a number of the outlet tubes. For these models a slightly different type of cuvette is used in which the liquid is introduced at the top and withdrawn at the bottom. 